\title{
Effect of Seed Pre-sowing Gamma-irradiation Treatment in Bread Wheat Lines Differing by Anthocyanin Pigmentation
}

\author{
E.I. Gordeeva ${ }^{1 * *}$, O.Yu. Shoeva ${ }^{1 * *}$, R.S. Yudina ${ }^{1}$, T.V. Kukoeva ${ }^{1}$ and E.K. KhlestKina ${ }^{1,2 *}$ \\ ${ }^{1}$ Institute of Cytology and Genetics, Siberian Branch of the Russian Academy of Sciences, \\ Lavrentjeva Ave. 10, Novosibirsk, 630090 Russia \\ ${ }^{2}$ Novosibirsk State University, Pirogova St. 2, Novosibirsk, 630090 Russia
}

(Received 6 December 2016; Accepted 4 July 2017;

Communicated by A. Börner and A. Goyal)

\begin{abstract}
Anthocyanins are natural antioxidants able to scavenge free radicals, which appear in plant cells under various environmental stresses. In wheat, anthocyanin pigments can be synthesized in vegetative and reproductive organs. The objective of the current study was to estimate the significance of these substances for wheat seedlings protection under irradiation stress (after treatment of dry seeds with moderate doses of gamma-irradiation, 50, 100 and $200 \mathrm{~Gy}$ ). For this goal a set of near-isogenic lines (8 NILs) carrying different combinations of the $P p$ (purple pericarp) and $R c$ (red coleoptile) alleles were used. The effect of gammairradiation on the growth parameters and anthocyanin content in coleoptiles was studied at the $4^{\text {th }}$ day after germination. The germination rate was not affected, while roots' and shoots' lengths and fresh weights as well as root number decreased significantly under irradiation treatment. The effect was deeper under higher doses. Irradiation treatment also induced change of root morphology ('hairy roots'). The effect of treatment on coleoptile anthocyanin content depended on allelic combination at the $R c$ loci. At the presence of 'weak' $R c-A 1$ allele anthocyanin content decreased, while it did not change in lines with $R c-A 1+R c-D 1$ combination (NILs with intensively colored coleoptiles). Factors 'pericarp color' and 'coleoptile color' influenced vigor of the seedlings under $50 \mathrm{~Gy}$, whereas under higher doses (100 and $200 \mathrm{~Gy}$ ) these factors did not contribute to growth parameters changes. Statistically significant positive effect of anthocyanins synthesized in coleoptile (in the presence of $R c-A 1+R c-D 1$ dominant alleles) on root growth of seedling germinated from 50 Gy-treated seeds was observed.
\end{abstract}

Keywords: anthocyanins, coleoptile color, gamma-irradiation, pericarp color, plant vigor, protective effect, $P p$ genes, $R c$ genes, seed germination, seedlings growth, Triticum aestivum L.

\section{Introduction}

The effects of gamma-irradiation include changes in the plant cellular structure and metabolism, e.g. dilation of thylakoid membranes, distortion and swelling of mitochondria and endoplasmic reticulum, alteration in photosynthesis, decrease of the relative water

\footnotetext{
*Corresponding author; E-mail: khlest@bionet.nsc.ru; Phone: +7(383)363-49-63; Fax: +7(383)333-12-78

**Authors contributed equally to this work
} 
content and the membrane integrity, modulation of the antioxidant systems, and accumulation of phenolic compounds and carotenoids (Kovács and Keresztes 2002; Kim et al. 2004; Wi et al. 2007; Abou-Zeid and Abdel-Latif 2014; Ramabulana et al. 2016). Generally, ionizing radiation may have different effects on plant metabolism, growth and reproduction depending on the dose: positive effects at very low doses (radiostimulation or radiation hormesis), detrimental consequences at intermediate levels and pronounced damage at high doses. Besides the dose, the severity of the effects is dependent on other factors such as species, cultivars, plant developmental stage (Arena et al. 2014).

The deleterious effects of ionizing radiation in biological systems arise directly via the interaction between radiation and target macromolecules or indirectly via the generation of free radicals, which are able to produce serious cell damage (Esnault et al. 2010). For scavenging free radicals plants have protective antioxidant system including antioxidant defense enzymes such as superoxide dismutase, catalase, and peroxidase and non-enzymatic antioxidants (Caverzan et al. 2016). Among non-enzymatic antioxidants pigmented flavonoid compounds anthocyanins can be considered. Their antioxidant capacity is almost four times higher than that one of ascorbic acid and $\alpha$-tocopherol (Bors et al. 1994; Wang et al. 1997). Accumulation of anthocyanins is positively related to plant adaptation under environment stresses such as salinity, drought, low temperature, heavy metals or UV radiation (Chalker-Scott 1999; Landi et al. 2015).

In bread wheat (Triticum aestivum L., BBAADD, $2 n=6 x=42$ ), anthocyanin pigments can be synthesized in vegetative and reproductive organs such as coleoptiles (controlled by the $R c$ genes), culms $(P c)$, leaf blades $(P l b)$, leaf sheaths $(P l s)$, glumes $(P g)$, auricles $(R a)$, grain pericarp (complementary genes $P p-1$ and $P p 3)$, and anthers (Pan) (Khlestkina 2012), but the significance of these substances for wheat plant protection has not been sufficiently estimated yet.

The objective of the current study was to investigate a protective role of the anthocyanin pigments accumulated in wheat pericarp and coleoptile against oxidative damages imposed by gamma-radiation. For this purpose a set of near-isogenic lines carrying different combinations of dominant and recessive alleles of the $P p$ and $R c$ genes were used.

\section{Materials and Methods}

\section{Plant material}

A set of near-isogenic lines (NILs) from the ICG collection of "GenAgro", differing in color of grain pericarp and coleoptile was used (Table 1). The lines have been developed previously in the Institute of Cytology and Genetics SB RAS (Novosibirsk, Russia) by Arbuzova et al. (1998) and Gordeeva et al. (2015) as a convenient model for studying anthocyanin regulatory network and for assessment of wheat anthocyanins' protective role and health benefits. The lines have been developed in the genetic background of spring bread wheat 'Saratovskaya 29' ('S29') having non-colored pericarp and light red coleoptile. Among the 'S29' NILs there are lines with trait combinations "non-colored pericarp + non-colored coleoptile", "light purple pericarp + light red coleoptile", "non- 
Table 1. The set of the NILs carrying various combinations of $P p$ alleles used in the current study

\begin{tabular}{|c|c|c|c|c|}
\hline No. & Line full name & Line short name & $\begin{array}{c}\text { Pericarp color } \\
\text { (dominant gene }(\mathrm{s}))\end{array}$ & $\begin{array}{l}\text { Coleoptile color } \\
\text { (dominant gene(s)) }\end{array}$ \\
\hline 1 & $\mathrm{i}: \mathrm{S} 29 p p-A 1 p p-D 1 p p 3$ & iJP7A & Non-colored & Non-colored \\
\hline 2 & $\mathrm{i}: \mathrm{S} 29 P p-A 1 p p-D 1 p p 3$ & S29 & Non-colored & Light red $(R c-A l)$ \\
\hline 3 & $\mathrm{i}: \mathrm{S} 29 P p-A 1 p p-D 1 P p 3 \mathrm{P}$ & iP2A & $\begin{array}{l}\text { Light purple } \\
(P p 3+P p-A 1)\end{array}$ & Light red $(R c-A l)$ \\
\hline 4 & $\mathrm{i}: \mathrm{S} 29 P p-A 1 p p-D 1 P p 3 \mathrm{PF}$ & iPF2A & $\begin{array}{l}\text { Light purple } \\
(P p 3+P p-A 1)\end{array}$ & Light red $(R c-A l)$ \\
\hline 5 & $\mathrm{i}: \mathrm{S} 29 P p-A 1 P p-D 1 p p 3 \mathrm{P}$ & iP7D & Non-colored & $\begin{array}{c}\text { Dark red } \\
(R c-A 1+R c-D 1)\end{array}$ \\
\hline 6 & $\mathrm{i}: \mathrm{S} 29 P p-A 1 P p-D 1 p p 3 \mathrm{PF}$ & iPF7D & Non-colored & $\begin{array}{c}\text { Dark red } \\
(R c-A 1+R c-D 1)\end{array}$ \\
\hline 7 & $\mathrm{i}: \mathrm{S} 29 P p-A 1 P p-D 1 P p 3 \mathrm{P}$ & iP & $\begin{array}{c}\text { Dark purple } \\
(P p 3+P p-A 1+P p-D 1)\end{array}$ & $\begin{array}{c}\text { Dark red } \\
(R c-A 1+R c-D 1)\end{array}$ \\
\hline 8 & i:S29Pp-A1Pp-D1Pp3PF & iPF & $\begin{array}{c}\text { Dark purple } \\
(P p 3+P p-A 1+P p-D 1)\end{array}$ & $\begin{array}{c}\text { Dark red } \\
(R c-A 1+R c-D l)\end{array}$ \\
\hline
\end{tabular}

Note: The lines development and their allelic combinations at the $R c$ and $P p$ loci were described in Gordeeva et al. 2015.

colored pericarp + dark red coleoptile", "dark purple pericarp + dark red coleoptile". The sets of dominant alleles determining phenotype of each line are described in Table 1.

\section{Irradiation treatment and measurements}

Dry seeds of the lines were irradiated with 50, 100 and 200 Gray (Gy), using Cesium-137 $\left({ }^{137} \mathrm{Cs}\right)$ gamma-irradiation source (IGUR-1", Institute Cytology and Genetic SB RAS, Novosibirsk, Russia). The treated and control seeds were placed in Petri dishes with filter paper moistened with distilled water and kept for 48 hours at $+4{ }^{\circ} \mathrm{C}$ in the darkness in a growth chamber «Rubarth Apparate» (RUMED GmbH) for synchronization of germination. Thereafter the temperature in the chamber was increased to $20^{\circ} \mathrm{C}$ and a daily cycle with $12 \mathrm{~h}$ light $/ 12 \mathrm{~h}$ darkness was set up.

At the fourth day after germination the length of the main root and that of the first leaf as well as fresh root and shoot weights, roots number and germination rate were measured. The experiment was performed in five replicates for each genotype and treatment, with twenty seedlings per replicate. Changes in root length were calculated as follows:

$$
\left(\frac{\text { root length under treatment }}{\text { root length without treatment }}-1\right) * 100 \%
$$

Changes in other root parameters and shoot lengths and weights, as well as OD530 values of anthocyanin extracts were calculated in the same way.

All experiments were carried out using seeds produced at the same time and in the same conditions. 


\section{Anthocyanin extraction}

For anthocyanin extraction, 20 frozen coleoptiles from each replicate were homogenized and incubated in $1 \% \mathrm{HCl} /$ methanol $\left(5 \mathrm{ml}\right.$ for $1 \mathrm{~g}$ of plant material) at $4{ }^{\circ} \mathrm{C}$ for 24 hours, then centrifuged at $12,000 \mathrm{~g}$ for $30 \mathrm{~min}$ at $4{ }^{\circ} \mathrm{C}$. After that supernatant was used for measurement of the relative anthocyanin content at $530 \mathrm{~nm}$ wavelength with a spectrophotometer «SmartSpec ${ }^{\mathrm{TM}}$ Plus» (BioRad).

\section{Statistical analysis}

The significance of differences between genetic stocks of control and stressed plants were assessed using nonparametric Mann-Whitney U-tests (Mann and Whitney 1947). The non-parametric Kruskal-Wallis one-way analysis of variance $H$-test (Kruskal and Wallis 1952) was used for determining the influence of gamma-irradiation on growth parameters of seedlings and factors 'pericarp color', 'coleoptile colour', and 'pericarp and coleoptile color' on growth parameters changes of wheat seedlings after seeds exposure to 50,100 and 200 Gy. The NILs were differently combined to test the effect of the coloration on growth parameters changes. In respect to pericarp color three groups were distinguished: 'non-colored' ('iJP7A', 'S29', 'iPF7D', ‘iP7D'), 'light purple' ('iPF2A', 'iP2A') and 'dark purple' ('iPF', 'iP'). According to the coleoptile color also three groups were distinguished: 'non-colored' ('iJP7A'), 'light red' ('S29', ‘iPF2A', 'iP2A') and 'dark red' ('iPF', ‘iP', 'iPF7D', 'iP7D'). Five groups of samples were distinguished with respect to pericarp and coleoptile color: 'non-colored pericarp + non-colored coleoptile' ('iJP7A'), 'non-colored pericarp + light red coleoptile' ('S29'), 'light purple pericarp + light red coleoptile' ('iPF2A', 'iP2A'), 'non-colored pericarp + dark red coleoptile' ('iPF7D', 'iP7D'), and 'dark purple pericarp + dark red coleoptile' ('iPF', 'iP'). Differences between the groups were tested by the Dunnett's post hoc test, taking $p \leq 0.05$ as the significance threshold. Spearman's rank correlation coefficients between the parameters were calculated. Statistical hypotheses were tested with the program Statistica 6.0 (StatSoft Inc., 2001).

\section{Results}

\section{Germination rate}

In control conditions, germination was 95-100\% (Table S1*). Gamma-irradiation seed treatment at different doses did not affect the germination rate (Table S2, Fig. 1) with the exception of 'iP2A' line under dose 200 Gy inducing 7\% decrease (Fig. 1).

\section{Shoot length and weight changes}

Irradiation treatment caused changes in shoot length (Fig. 2A, Tables S2-S3). The shoot length decrease varied after $50 \mathrm{~Gy}$ from 29.7 (in the line 'iPF') to $44.1 \%$ ('iP2A'), after

\footnotetext{
*Further details about the Electronic Supplementary Material (ESM) can be found at the end of the article.
} 


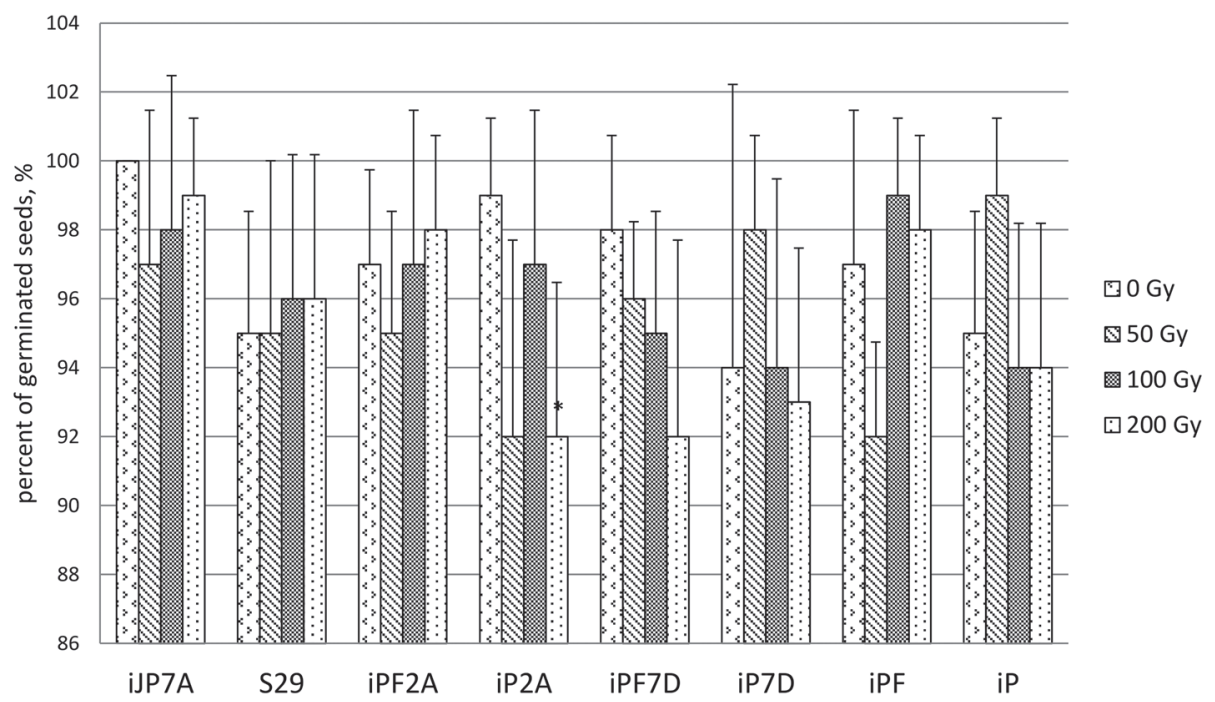

Figure 1. Effect of gamma radiation on germination rate of wheat seeds exposed to 0, 50, 100 and $200 \mathrm{~Gy}$. $*$ differences between treated (50,100 and $200 \mathrm{~Gy})$ and control ( $0 \mathrm{~Gy}$ ) samples were significant at $p \leq 0.05$

100 Gy from 50.2 ('iPF') to $58.2 \%$ ('iP7D'), and from 55.4 ('iPF') to $61.2 \%$ ('iP7D') (Fig. 2A).

The shoot weight was also influenced by gamma-irradiation (Fig. 2B, Tables S2, S4). The shoot weight decrease was within 30.5 ('iPF') - 46.3\% ('iJP7A'), 59.7 ('iP2A') 64.6\% ('iPF7D'), 64.6 ('iPF2A') - 68.6\% ('iP7D'), after 50, 100 and 200 Gy, respectively (Fig. 2B).

The level of shoot length and weight decrease varied notably between the lines after 50 Gy treatment, whereas after higher doses the changes of the parameters were similar in all lines (Figs 2A, 2B).

There was low positive correlation between shoot length of control plants and extent of the shoot length decrease after seed exposure $\left(r_{\mathrm{s}}=0.18, p \leq 0.05\right)$, whereas there was no correlation between shoot weight of control plants and extent of the shoot weight decrease.

\section{Root system changes}

Irradiation treatment caused significant changes in root systems, e.g. the length and weight of the main root, the number of roots and the percent of seedlings with hairy roots (Tables S2, S5-S8).

The root length decrease after seed irradiation by 50, 100, and 200 Gy ranged from 46.1 ('iPF') to $67.6 \%$ ('iJP7A'), from 65.2 ('iPF') to 72.8 ('iJP7A'), and from 69.1 ('iP2A') to 76.3 ('iJP7A'), respectively (Fig. 2C). 

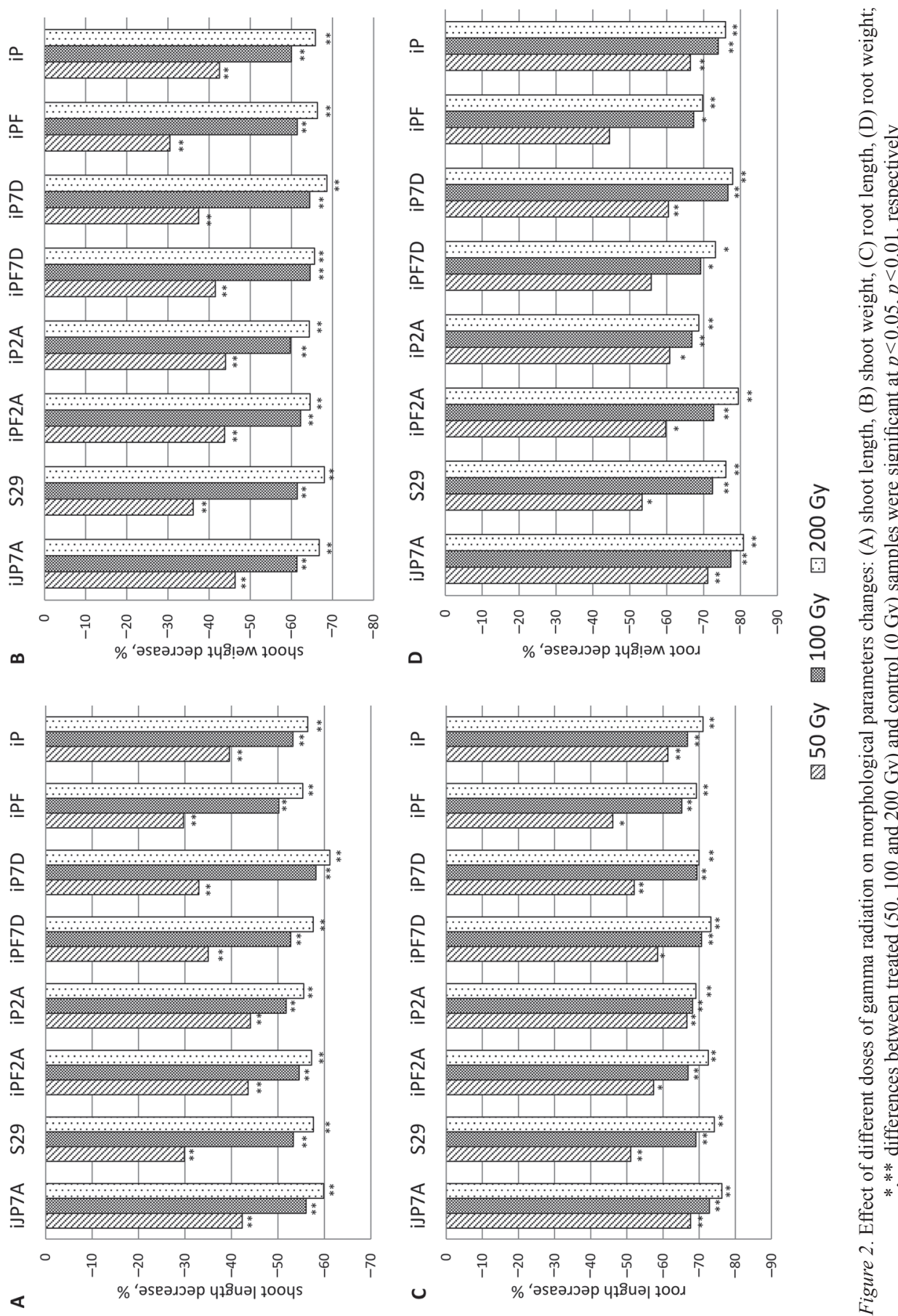

Cereal Research Communications 46, 2018 
Decreasing of the root weight was from 44.5 ('iPF') to $71.1 \%$ (' $\mathrm{iJP7A}$ '), from 66.8 ('iP2A') to $77.4 \%$ ('iJP7A') and from 68.7 ('iP2A') to $80.8 \%$ ('iJP7A') after 50, 100 and 200 Gy, respectively (Fig. 2D).

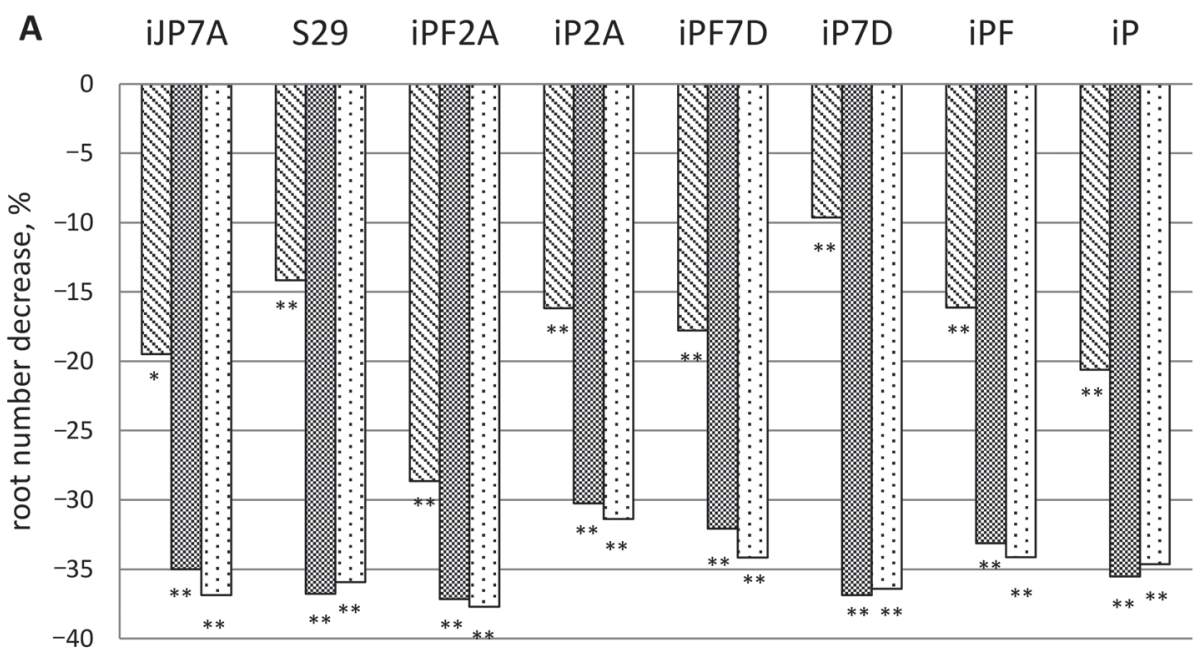

B

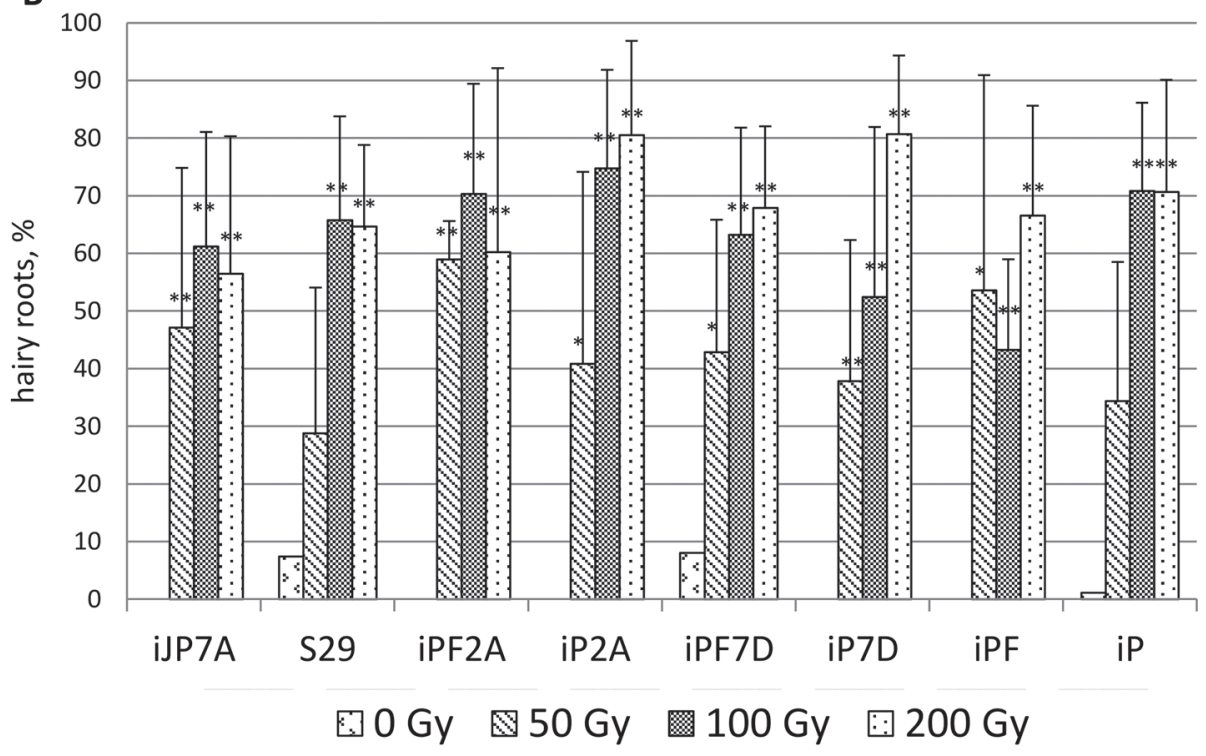

Figure 3. Effect of different doses of gamma radiation on (A) changes of roots number and (B) percent of seedlings with hairy roots. The data are present as mean of five replicates \pm SD. *** differences between treated $(50,100$ and $200 \mathrm{~Gy})$ and control ( $0 \mathrm{~Gy})$ were samples significant at $p \leq 0.05, p \leq 0.01$, respectively 
There were low positive correlations between root length and weight of control plants and extent of the root length and weight reduction under treatment (for root length $r_{s}=0.29, p \leq 0.05$; for root weight $r_{s}=0.37, p \leq 0.05$ ).

The control plants root number varied from $4.67 \pm 0.21$ ('iPF7D') to $4.93 \pm 0.12$ ('iJP7A') (Table S7). Root number decreased after treatment. Decreasing of the root number varied within 9.6 ('iP7D') -28.6\% ('iPF2A'), 30.2 ('iP2A') -37.1\% ('iPF2A'), 31.4 ('iP2A') - 37.7\% ('iPF2A'). There was no correlation between root number in control and changes of root number under treatment.

In control plants, only several seedlings with hairy roots were noted in 'S29', 'iPF7D', and 'iP', whereas root hairs appeared in all lines under the influence of gamma-irradiation (Table S8). The most noticeable percent of seedlings with hairy roots was observed in 'iPF2A' $(+59 \%)$, in 'iP2A' $(+75 \%)$, and in 'iP7D' $(+81 \%)$, while the lowest one was in 'S29' $(+29 \%)$, in 'iPF' $(+43 \%)$, in 'iJP7A' $(+56 \%)$, under 50, 100, and $200 \mathrm{~Gy}$, respectively (Fig. 3B). The differences between lines were not statistically significant, except for 'iP2A' vs 'iPF' and 'iPF' vs 'iP' under 100 Gy (Table S8).

\section{Changes of relative anthocyanin content}

Gamma-irradiation changed anthocyanin content in coleoptiles (Tables S2, S9). The genotypes with light red coleoptile pigmentation ('S29', 'iPF2A', 'iP2A'), predetermined by the 'weak' $R c-A 1$ allele, demonstrated significant dose-dependent decrease in the relative content of anthocyanins. The most pronounced decrease of level of anthocyanins was observed in 'iP2A' (-33.1\%) after $50 \mathrm{~Gy}$, in 'iPF2A' (-41.4\%) after $100 \mathrm{~Gy}$, and in 'S29' $(-45.6 \%)$ after $200 \mathrm{~Gy}$. In the lines with dark red coleoptile color ('iPF7D', 'iP7D', 'iPF', 'iP') conferred by the combination of $R c-A 1$ with 'strong' $R c-D 1$ allele, the changes between control and treated samples were not statistically significant (Fig. 4).

Strong negative correlations were observed between anthocyanin content in coleoptile and gamma-irradiation doses in group of genotypes with lightly colored coleoptiles $\left(r_{s}=-0.78, p \leq 0.05\right)$, whereas no correlation was found between those parameters in group of genotypes with intensively colored coleoptiles $\left(r_{s}=-0.15, p>0.05\right)$.

Overall, it can be concluded that anthocyanin biosynthesis in different genotypes responds differently on irradiation in dependence on alleles of the $R c-1$ genes predetermining the intensity of anthocyanin pigmentation.

\section{Effect of coleoptile color, pericarp color, and their combinations on changing growth parameters of wheat seedlings under irradiation treatment}

The one-way ANOVA on ranks was run to evaluate the effect of coleoptile and pericarp color, and combination of these factors on growth parameters changes of wheat seedlings after dry grain treatment with moderate doses of gamma-irradiation. As summarized in Tables S10-S22 these factors affect morphological parameters changes only under 50 Gy, whereas under higher doses the factors have no effect on growth parameters changes. 


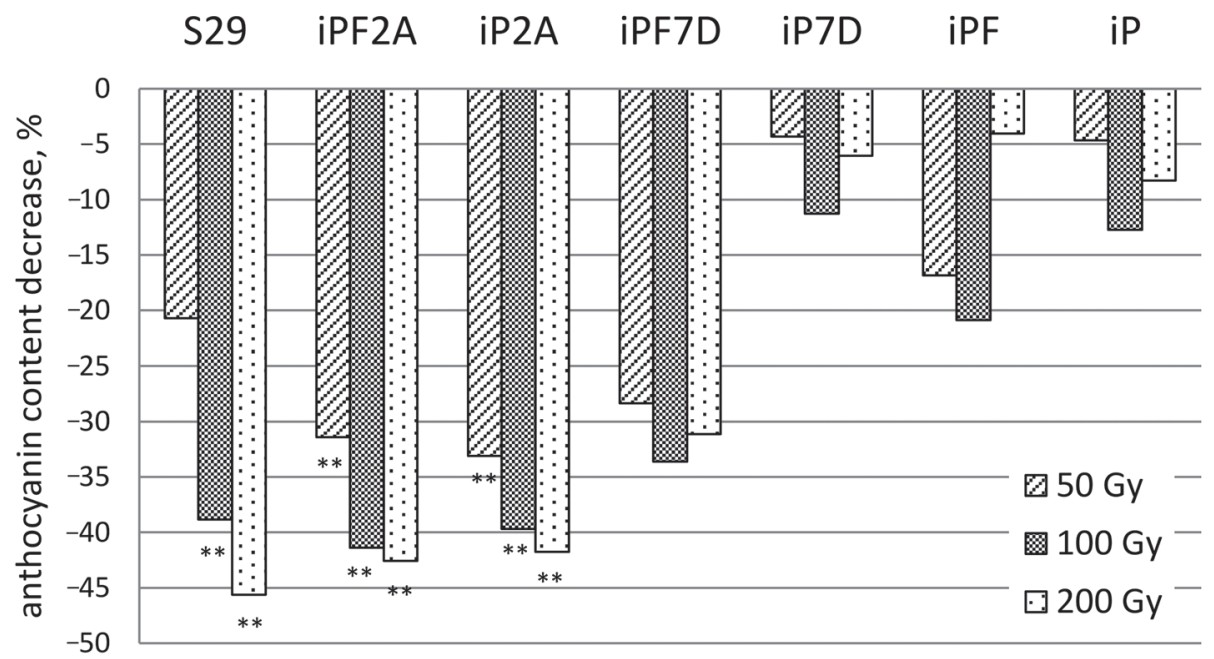

Figure 4. Changes in relative anthocyanin content $\left(\mathrm{OD}_{530}\right)$ in coleoptiles of seedlings germinated from 50, 100 and 200 Gy exposed seeds relative to that in control experiment ( 0 Gy). *** differences between treated and control samples were significant at $p \leq 0.05, p \leq 0.01$, respectively

The notable positive effect of anthocyanin coloration on vigor of seedling germinated from 50 Gy-treated seeds was observed in the group of samples having dark red coleoptile (Fig. S1A-C). This group was characterized with the lowest reduction of root length (Fig. S1B). The similar tendency was with changes in shoot length (Fig. S1A) and root weight (Fig. S1C).

The root length decrease did not vary among the groups of the genotypes with darkcolored or non-colored pericarp, whereas the group of the samples with light-colored pericarp had the highest reduction of shoot length (Fig. S1D).

In respect to combination of pericarp and coleoptile color, the highest reduction of shoot and root length under 50 Gy treatment was in non-colored 'iJP7A', however, in case of root length the difference was not statistically significant (Fig. S1E-F).

The obtained results demonstrated protective effect of coleoptile pigmentation on growth parameters of wheat seedlings germinated from 50 Gy-treated seeds, whereas under higher doses (100 and $200 \mathrm{~Gy}$ ) anthocyanins did not contribute to growth parameters changes.

\section{Discussion}

\section{Response of flavonoid metabolism to irradiation treatment}

Ionizing radiation generally affects flavonoid biosynthesis and related genes (Nagata et al. 2003; Hong et al. 2014; Ramabulana et al. 2016). Studying the effect of irradiation on anthocyanin metabolism in leaves, Sparrow et al. (1968) revealed an increase of the an- 
thocyanin content in 35 different plant species and concluded that increase of anthocyanin biosynthesis is a general reaction of plants on ionizing radiation. The authors noted that irradiation doses, which induce anthocyanin content increase, are species-specific. They varied from 30 Gy in Acer saccharum to 3420 Gy in Luzula acuminata.

This stimulating effect of ionizing radiation on anthocyanin metabolism was detected after exposure of seedlings and adult plants. Opposite tendency was observed when seeds were treated. In these cases, the post-irradiation suppression of flavonoid synthesis has been observed (Karabanov and Veremeitschik 1972; Zhu et al. 2010; Mohajer et al. 2014). Response of flavonoid metabolism to irradiation has also tissue-specific features (Margna and Vainjärv 1976).

In the current study, the dry wheat seeds irradiation treatment also caused a reduction of anthocyanin content in coleoptiles germinated from treated seeds compared to untreated control (Fig. 4). However, the response was genotype-dependent: in NILs having Rc-A1 ('S29', 'iPF2A', 'iP2A'), predetermining light red coleoptile color a significant decrease of anthocyanin content was observed, whereas in NILs with additional 'strong' $R c-D 1$ allele ('iPF7D', 'iP7D', 'iPF', 'iP') conferring dark red color, there was just a tendency of reduced anthocyanin synthesis, which was statistically insignificant (Fig. 4).

So, in the current study, it was for the first time shown that in addition to previously demonstrated dose-, species- and tissues-specificity of flavonoid biosynthesis response on irradiation treatment, there are genotype-dependent effects of gamma-irradiation treatment on the changes of anthocyanin content.

\section{Protective role of anthocyanin biosynthesis genes under irradiation treatment}

In spite of many studies showing response of anthocyanin synthesis on gamma-irradiation treatment, information demonstrating protective effect of these substances under such type of stress was absent. Here, comparison of precise genetic stocks such as nearisogenic lines differing by combinations of anthocyanin biosynthesis regulatory alleles allowed investigating a role of these genes in protection of seedlings germinated from seeds treated with moderate doses (50,100 and $200 \mathrm{~Gy})$. Under higher doses (100 and $200 \mathrm{~Gy}$ ) there was none protective effect, while under 50 Gy the positive effect of the coleoptiles' pigmentation on growth parameters was detected (Fig. S1A-C). At the same time anthocyanins accumulated in pericarp before treatment appeared to be useful for seedling protection neither under higher doses (100 and 200 Gy; Table S10) nor under lowest dose 50 Gy (Fig. S1D-F; Table S10). Different effect of coleoptile and pericarp coloration may have two explanations. The first hypothesis is related to importance of tissue location of protective substances anthocyanins. The second hypothesis considers importance of some processes taking place during anthocyanin synthesis (or significance of other non-colored substances produced along with anthocyanins). In coleoptiles, these processes are ongoing during seedling growth after dry seed treatment, while in pericarp just previously synthesized anthocyanins present.

In the current study, a protective role of anthocyanins accumulated in wheat coleoptile (but not in grain) against gamma-radiation was demonstrated by using the precise genetic 
model (a set of near-isogenic lines differing by allelic state of the genes conferring pigmentation). Although the anthocyanin content in seedlings germinated from the treated seeds was not changed or even decreased, the lines with intensively colored coleoptile were more tolerant to irradiation stress in comparison with their non-colored sister line. This protective effect was observed only under low dose of irradiation (50 Gy) whereas under higher doses (100 and $200 \mathrm{~Gy}$ ) anthocyanins seemed to be not enough effective as protective compounds. The observation of a positive role of anthocyanin accumulated in coleoptiles on vigor of seedlings germinated from 50 Gy-treated wheat grains can be related with anthocyanins' free radicals scavenging capacity.

\section{Acknowledgements}

This work was supported by the Russian Science Foundation (project 16-14-00086). Growing of wheat plants in ICG Plant Growth Core Facility was supported by the ICG project 0324-2016-001. We thank Ms Galina Generalova and Mrs Olga Zakharova for technical assistance and Dr Elena Antonova for fruitful discussion.

\section{References}

Abou-Zeid, H.M., Abdel-Latif, S.A. 2014. Effects of gamma irradiation on biochemical and antioxidant defense system in wheat (Triticum aestivum L.) seedlings. Int. J. Adv. Res. 2:287-300.

Arbuzova, V.S., Maystrenko, O.I., Popova, O.M. 1998. Development of near-isogenic lines of the common wheat cultivar 'Saratovskaya 29'. Cereal Res. Commun. 26:39-46.

Arena, C., De Micco, V., Macaeva, E., Quintens, R. 2014. Space radiation effects on plant and mammalian cells, and mammalian cells. Acta Astronautica 104:419-431. http://dx.doi.org/10.1016/j.actaastro.2014.05.005

Bors, W., Mochel, C., Saran, M. 1994. Flavonoid antioxidants: Rate constants for reactions with oxygen radicals. Met. Enzymol. 234:420-429.

Caverzan, A., Casassola, A., Patussi Brammer, S.P. 2016. Reactive oxygen species and antioxidant enzymes involved in plant tolerance to stress. In: Shanker, A. (ed.), Abiotic and Biotic Stress in Plants - recent advances and future perspectives. InTech. Rijeka, Croatia. pp. 463-480.

Chalker-Scott, L. 1999. Environmental significance of anthocyanins in plant stress responses. Photochem. Photobiol. 70:1-9.

Esnault, A.M., Legue, F., Chenal, C. 2010. Ionizing radiation: advances in plant response. Environ. Exp. Bot. 68:231-237.

Gordeeva, E.I., Shoeva, O.Y., Khlestkina, E.K. 2015. Marker-assisted development of bread wheat near-isogenic lines carrying various combinations of purple pericarp $(P p)$ alleles. Euphytica 203:469-476.

Hong, M.J., Kim, J.B., Yoon, Y.H., Kim, S.H., Ahn, J.W., Jeong, I.Y., Kang, S.Y., Seo, Y.W., Kim, D.S. 2014. The effects of chronic gamma irradiation on oxidative stress response and the expression of anthocyanin biosynthesis-related genes in wheat (Triticum aestivum). Int. J. Radiat. Biol. 17:1-31.

Khlestkina, E.K. 2012. Genes determining coloration of different organs in wheat. Vavilovskii Zhurnal Genetiki i Selektsii (Vavilov J. of Genetics and Breeding) 16:202-216.

Karabanov, I.A., Veremeitschik, V.Ye. 1972. The influence of irradiation on the productivity and the content of polyphenols in buckwheat. Dokl. AN SSSR. 203:488-490. (in Russian)

Kim, J. H., Baek, M.H., Chung, B.Y., Wi, S.G., Kim, J.S. 2004. Alterations in the photosynthetic pigments and antioxidant machineries of red pepper (Capsicum annuum L.) seedlings from gamma-irradiated seeds. J. Plant Biol. 47:314-321.

Kovács, E., Keresztes, Á. 2002. Effect of gamma and UV-B/C radiation on plant cells. Micron. 3:199-210. 
Kruskal, W.H., Wallis W.A. 1952. Use of ranks in one-criterion variance analysis. J. Am. Stat. Assoc. 47:583621.

Landi, M., Tattini, M., Gould, K.S. 2015. Multiple functional roles of anthocyanins in plant-environment interactions. Environ. Exp. Bot. 119:4-17.

Mann, H.B., Whitney, D.R. 1947. On a test of whether one of two random variables is stochastically larger than the other. Ann. Math. Statist. 18:50-60.

Margna, U., Vainjärv, T. 1976. Irradiation effects upon flavonoid accumulation in buckwheat seedlings. Environ. Exp. Bot. 16:201-208.

Mohajer, S., Taha, R.M., Lay, M.M., Esmaeili, A.K., Khalili, M. 2014. Stimulatory effects of gamma irradiation on phytochemical properties, mitotic behaviour, and nutritional composition of sainfoin (Onobrychis viciifolia Scop.). Sci. World. J. Article ID 854093:1-9. http://dx.doi.org/10.1155/2014/854093.

Nagata, T., Todoriki, S., Masumizu, T., Suda, I., Furuta, S., Du, Z., Kikuchi, S. 2003. Levels of active oxygen species are controlled by ascorbic acid and anthocyanin in Arabidopsis. J. Agric. Food. Chem. 51:29922999.

Ramabulana, T., Mavunda, R.D., Steenkamp, P.A., Piater, L.A., Dubery, I.A., Madala, N.E. 2016. Perturbation of pharmacologically relevant polyphenolic compounds in Moringa oleifera against photo-oxidative damages imposed by gamma radiation. J. Photochem. Photobiol. B. 156:79-86.

Sparrow, A.H., Furuya, M., Schwemmer, S.S. 1968. Effects of X- and gamma radiation on anthocyanin content in leaves of Rumex and other plant genera. Rad. Bot. 8:7-16.

Wang, H., Cao, G., Prior, R.L. 1997. Oxygen radical absorbing capacity of anthocyanins. J. Agric. Food Chem. 45:304-309.

Wi, S.G., Chung, B.Y., Kim, J.S., Kim, J.H., Baek, M.H., Lee, J.W., Kim, Y.S. 2007. Effects of gamma irradiation on morphological changes and biological responses in plants. Micron. 38:553-564.

Zhu, F., Cai, Y.Z., Bao, J., Corke, H. 2010. Effect of $\gamma$-irradiation on phenolic compounds in rice grain. Food Chem. 120:74-77.

\section{Electronic Supplementary Material (ESM)}

Electronic Supplementary Material (ESM) associated with this article can be found at the website of CRC at http://www.akademiai.com/content/120427/

Electronic Supplementary Table S1. The percent of germinated seeds on day 4 (mean of 5 replicates \pm SD, \%)

Electronic Supplementary Table S2. Kruskal-Wallis $H$-test: effect of gamma irradiation on morphological parameters of wheat seedlings

Electronic Supplementary Table S3. Shoot length (mean of 5 replicates \pm SD, mm)

Electronic Supplementary Table S4. Shoot weight (mean of 5 replicates \pm SD, mg)

Electronic Supplementary Table S5. Root length (mean of 5 replicates \pm SD, mm)

Electronic Supplementary Table S6. Root weight (mean of 5 replicates \pm SD, mg)

Electronic Supplementary Table S7. Root number (mean of 5 replicates \pm SD)

Electronic Supplementary Table S8. The percentage of seedlings with hairy roots (mean of 5 replicates \pm SD)

Electronic Supplementary Table S9. The relative anthocyanin content (OD530) in coleoptiles of lines with dominant $R c$ genes (mean of 5 replicates $\pm \mathrm{SD}$ ) 
Electronic Supplementary Table S10. The summary of the Kruskal-Wallis $H$-test analysis, evaluating effect of the factors 'grain color', 'coleoptile color', and both of the factors on morphological parameters of wheat seedlings grown from irradiated seeds. The preliminary data on Kruskal-Wallis $H$-test analysis are presented in Tables S11-S22

Electronic Supplementary Table S11. Kruskal-Wallis $H$-test analysis: effect of pericarp color on growth parameters of wheat seedlings in control samples (0 Gy)

Electronic Supplementary Table S12. Kruskal-Wallis $H$-test analysis: effect of pericarp color on growth parameters of wheat seedlings under $50 \mathrm{~Gy}$

Electronic Supplementary Table S13. Kruskal-Wallis $H$-test analysis: effect of pericarp color on growth parameters of wheat seedlings under $100 \mathrm{~Gy}$

Electronic Supplementary Table S14. Kruskal-Wallis $H$-test analysis: effect of pericarp color on growth parameters of wheat seedlings under $200 \mathrm{~Gy}$

Electronic Supplementary Table S15. Kruskal-Wallis $H$-test analysis: effect of coleoptile color on growth parameters of wheat seedlings in control samples

Electronic Supplementary Table S16. Kruskal-Wallis $H$-test analysis: effect of coleoptile colour on growth parameters of wheat seedlings under $50 \mathrm{~Gy}$

Electronic Supplementary Table S17. Kruskal-Wallis $H$-test analysis: effect of coleoptile colour on growth parameters of wheat seedlings under $100 \mathrm{~Gy}$

Electronic Supplementary Table S18. Kruskal-Wallis $H$-test analysis: effect of coleoptile colour on growth parameters of wheat seedlings under $200 \mathrm{~Gy}$

Electronic Supplementary Table S19. Kruskal-Wallis $H$-test analysis: effect of pericarp and coleoptile color on growth parameters of wheat seedlings in control samples

Electronic Supplementary Table S20. Kruskal-Wallis $H$-test analysis: effect of pericarp and coleoptile color on growth parameters of wheat seedlings under $50 \mathrm{~Gy}$

Electronic Supplementary Table S21. Kruskal-Wallis $H$-test analysis: effect of pericarp and coleoptile color on growth parameters of wheat seedlings under $100 \mathrm{~Gy}$

Electronic Supplementary Table S22. Kruskal-Wallis $H$-test analysis: effect of pericarp and coleoptile color on growth parameters of wheat seedlings under $200 \mathrm{~Gy}$

Electronic Supplementary Figure S1. Effect of coleoptile color (A-C), pericarp color (D), and their combinations $(\mathrm{E}-\mathrm{F})$ on changing growth parameters of wheat seedlings under 50 Gy treatment. The data are presented as mean in the group $\pm \mathrm{SD}$. The same letters indicate significantly undistinguished groups of samples $(p>0.05$, Dunnett's post hoc test) 